\title{
Article
}

\section{In Vitro and In Vivo Biocompatibility of Boron/Nitrogen Co-Doped Carbon Nano-Onions}

\author{
Marta d'Amora ${ }^{1,+}\left(\mathbb{D}\right.$, Adalberto Camisasca ${ }^{2,+}{ }^{\text {, Raul Arenal }}{ }^{3,4,5}$ (D) and Silvia Giordani $^{1,2, *(D)}$ \\ 1 Nano Carbon Materials, Istituto Italiano di Tecnologia (IIT), 16163 Genoa, Italy; marta.damora@iit.it \\ 2 School of Chemical Sciences, Dublin City University, Glasnevin, Dublin 9, Ireland; \\ adalberto.camisasca@dcu.ie \\ 3 Instituto de Nanociencia y Materiales de Aragon (INMA), CSIC-U. de Zaragoza, 50009 Zaragoza, Spain; \\ arenal@unizar.es \\ 4 Laboratorio de Microscopias Avanzadas (LMA), Universidad de Zaragoza, 50018 Zaragoza, Spain \\ 5 ARAID Foundation, 50018 Zaragoza, Spain \\ * Correspondence: silvia.giordani@dcu.ie \\ + These authors contributed equally to this work.
}

Citation: d'Amora, M.; Camisasca,

A.; Arenal, R.; Giordani, S. In Vitro and In Vivo Biocompatibility of Boron/Nitrogen Co-Doped Carbon Nano-Onions. Nanomaterials 2021, 11 , 3017. https://doi.org/10.3390/ nano11113017

Academic Editors: Saura Sahu

Received: 28 September 2021 Accepted: 6 November 2021 Published: 10 November 2021

Publisher's Note: MDPI stays neutral with regard to jurisdictional claims in published maps and institutional affiliations.

Copyright: (c) 2021 by the authors. Licensee MDPI, Basel, Switzerland. This article is an open access article distributed under the terms and conditions of the Creative Commons Attribution (CC BY) license (https:/ / creativecommons.org/licenses/by/ $4.0 /)$.

\begin{abstract}
Boron/nitrogen, co-doped, carbon nano-onions (BN-CNOs) have recently shown great promise as catalysts for the oxygen reduction reaction, due to the improved electronic properties imparted by the dopant atoms; however, the interactions of BN-CNOs with biological systems have not yet been explored. In this study, we examined the toxicological profiles of BN-CNOs and oxidized $\mathrm{BN}-\mathrm{CNOs}$ (oxi-BN-CNOs) in vitro in both healthy and cancer cell lines, as well as on the embryonic stages of zebrafish (Danio rerio) in vivo. The cell viabilities of both cell lines cells were not affected after treatment with different concentrations of both doped $\mathrm{CNO}$ derivatives. On the other hand, the analysis of $\mathrm{BN}-\mathrm{CNO}$ and oxidized $\mathrm{BN}-\mathrm{CNO}$ interactions with zebrafish embryos did not report any kind of perturbations, in agreement with the in vitro results. Our results show that both doped CNO derivatives possess a high biocompatibility and biosafety in cells and more complex systems.
\end{abstract}

Keywords: carbon nano-onion; heteroatom doping; cells; zebrafish; biosafety

\section{Introduction}

Carbon nano-onions (CNOs) are a member of the carbon family consisting of a multi nested fullerene-like structure [1] and a size ranging from a few to tens of nm depending on the synthetic methodology [2]. In the last decade, CNOs have shown great promise in bio-related applications due to their small size, high surface area, and easy surface modification approaches, as well as low in vitro cytotoxicity $[3,4]$ and excellent in vivo biocompatibility $[4,5]$. In particular, their remarkable emission properties, high cellular uptake, and low cytotoxicity of different fluorescent-labelled CNOs allowed their use as bioimaging probes in several cell lines [3,6,7]. In addition, compared to other fluorescent nanoparticles that require multi-step synthesis protocols, CNOs can be produced by the thermal annealing of detonation nanodiamonds, which ensure a low-cost, single-step, and high-yield way to achieve nanoparticle synthesis [8,9].

Furthermore, the high conductivity and chemical stability, together with the development of novel conjugation protocols with biologically relevant molecules, made this material very attractive for biosensing applications, showing an enhanced analytical performance in terms of sensitivity and limit of detection [10-13].

Recently, we reported a new member of the CNO family, namely a boron/nitrogen co-doped derivative (BN-CNOs), and demonstrated how the simultaneous introduction of both boron and nitrogen atoms was able to confer remarkable catalytic properties to the CNO materials towards the oxygen reduction reaction [14]. However, the biological behavior of this novel class of CNOs has not yet been explored. In addition, the presence 
of dopants, such as boron and nitrogen, may impact and change to some extent the biocompatibility of the CNOs. Considering that carbon nanomaterials employed in any application may result in human and environmental exposure [15-17], with a consequent potential risk of adverse effects, it is necessary to fully assess the toxicological profile of $\mathrm{BN}-\mathrm{CNO}$. To this end, in this work, we evaluated for the first time the toxicity of BN-CNOs both in vitro and in vivo.

In addition, we decorated the $\mathrm{CNO}$ surface with oxygen-containing groups via an oxidation procedure in order to enhance the water solubility of the constructs, opening the way for further functionalization procedures with biologically relevant molecules.

$\mathrm{BN}-\mathrm{CNO}$ and their oxidized counterparts (oxi-BN-CNOs) were characterized by highresolution transmission electron microscopy (HRTEM), electron energy loss spectroscopy (EELS), X-ray photoelectron spectroscopy (XPS), UV-Vis absorption spectroscopy, dynamic light scattering (DLS), attenuated total reflectance Fourier transform infrared spectroscopy (ATR-FTIR) and thermogravimetric analysis (TGA).

The potential in vitro cytotoxicity of the CNO materials was assessed on two different cell lines. For our investigation, we selected mouse embryonic fibroblast cells (NIH 3T3) and human breast adenocarcinoma cells (MCF7) as healthy and cancer cell lines, respectively. Even if these cellular screenings provided preliminary results on the nanotoxicity of the doped $\mathrm{CNO}$ derivatives, we decided to analyze in more detail their possible dose-dependent effects in vivo in zebrafish (Danio rerio).

Nowadays, zebrafish are a valuable and alternative animal model since their embryos and larvae are not considered animals until 5 days post fertilization (dpf), in accordance with the EU legislation [18]. Zebrafish are commonly used for nanotoxicity assessments due to their unique features, such as a short reproduction time, small size, and optical transparency, that allow for high-throughput assessments. Furthermore, they have a significant anatomical, physiological, and genomic homology to other higher vertebrates [19-24].

In line with previous studies, where zebrafish were successfully employed for evaluating the toxicity of several carbon nanomaterials [25], we explored the toxicological profile of the doped CNO derivatives in zebrafish (Danio rerio) using a 5-day embryonic assay. We analyzed the biological responses of both zebrafish embryos and developing larvae treated with BN-CNOs and oxi-BN-CNOs, in terms of mortality/survival rate, swimming (frequency of movements), cardiac (heart rate) activities, and hatching success.

\section{Materials and Methods}

\subsection{Materials}

All reagents and solvents were purchased from Merck (Dublin, Ireland). Detonation nanodiamonds (DNDs) were bought from Carbodeon Ltd. (Vantaa, Finland). All materials were used as received.

\subsection{Material Synthesis and Characterization}

Boron/nitrogen carbon nano-onions (BN-CNOs) were produced through a previously reported thermal annealing process utilizing DNDs and boric acid $\left(\mathrm{H}_{3} \mathrm{BO}_{3}\right)$ as precursors [14]. The synthesis of oxidized BN-CNOs (oxi-BN-CNOs) was accomplished following a chemical oxidative procedure using $3 \mathrm{M}$ nitric acid $\left(\mathrm{HNO}_{3}\right)$ as oxidizing agent [3].

The local composition and the structure of the $\mathrm{CNO}$ materials were investigated via high-resolution transmission electron microscopy (HRTEM) (FEI-Thermo Fisher Scientific, Eindhoven, The Netherland). The HRTEM samples were prepared by dispersing a drop of the ultrasonicated suspension in ethanol onto carbon holey-supported $\mathrm{Cu}$ grids. We used two different aberrations-corrected FEI Titan microscopes, both operated at $80 \mathrm{kV}$. For HRTEM, a high-resolution, Cs-corrected FEI Titan-Cube was employed. Electron energy loss spectroscopy (EELS) measurements were developed using an FEI Titan Low-Base microscope (FEI-Thermo Fisher Scientific, Eindhoven, The Netherland) equipped with a Cs probe corrector, a monochromator, and ultra-bright X-FEG electron source. The convergence angle was $25 \mathrm{mrad}$, the energy resolution was $\sim 1 \mathrm{eV}$ and the convergence 
and collection angles were 25 and 50 mrad, respectively. XPS analyses were carried out on a PHI-5000 VersaProbe spectrometer (Physical Electronics Inc. (PHI), Chanhassen, $\mathrm{MN}, \mathrm{USA}$ ) equipped with a monochromatic Al $\mathrm{K} \alpha$ radiation source. XPS data processing was performed by using Multipak software (Version 9.7, Physical Electronics Inc. (PHI), Chanhassen, MN, USA) with energy calibration performed with respect to the $C 1$ s peak. TGA analyses were performed on a Netzsch TG 209 F1 Libra thermogravimetric analyzer (NETZSCH-Gerätebau GmbH, Weimar, Germany) by heating up the samples and placing them in an $\mathrm{Al}_{2} \mathrm{O}_{3}$ pan from $\mathrm{RT}$ to $900{ }^{\circ} \mathrm{C}$ at $10{ }^{\circ} \mathrm{C} / \mathrm{min}$ under a constant airflow. ATR-FTIR analyses were carried out on solid samples on a Perkin Elmer Spectrum 100 (Perkin Elmer, Dublin, Ireland) under air conditions in the $3000-700 \mathrm{~cm}^{-1}$ range. UV-Vis absorption spectra were acquired with a Shimadzu UV-1800 spectrophotometer (Shimadzu, Milton Keynes, UK) by using standard quartz cuvettes with a $10 \mathrm{~mm}$ path length. DLS analyses were carried out on a Malvern Nano-ZS instrument (Malvern Panalytical, Dublin, Ireland) operating in backscattering $\left(173^{\circ}\right)$ mode, with an automatic selection of the number of independent measurements and the optimal detector position. For absorption and DLS analyses, the CNO materials were dispersed via ultrasonication in deionized water at $1 \mathrm{mg} / \mathrm{mL}$ and then diluted to the desired final concentrations. Prior to the analyses, each solution was sonicated for an additional $10 \mathrm{~min}$.

\subsection{In Vitro Biological Methods \\ Cell Culture}

Mouse embryonic fibroblast cells (NIH/3T3, ATCC CL173 passage number 3) and human breast adenocarcinoma cells (MCF7, ATCC HTB22, passage number 3) were cultured in Dulbecco's modified Eagle's medium (DMEM) (Thermo Fisher Scientific, Inc., Waltham, MA, USA), supplemented with 10\% fetal bovine serum (FBS) (Thermo Fisher Scientific, Inc., Waltham, MA, USA), $100 \mathrm{IU} / \mathrm{mL}$ penicillin, and $100 \mu \mathrm{g} / \mathrm{mL}$ streptomycin (Thermo Fisher Scientific, Inc., Waltham, MA, USA) at $37^{\circ} \mathrm{C}$, in a humidified $5 \% \mathrm{CO}_{2}$ atmosphere.

For the toxicity assessment, cells $\left(1 \times 10^{4}\right.$ cells/well $)$ were seeded in 96-well plates. Following overnight incubation, the medium was removed and replaced with fresh DMEM, containing various concentrations $(0.5,1,5$, and $10 \mu \mathrm{g} / \mathrm{mL})$ of BN-CNOs and oxi-BNCNOs for $72 \mathrm{~h}$. Cells were incubated with 5\% dimethyl sulfoxide (DMSO is toxic to any kind of cells) as a positive control, while cells with the fresh medium were used as a negative control. The cytotoxicity was assessed using WST-1 assay (Roche Applied Science, Mannheim, Germany), according to our previous report [4]. This simple colorimetric assay was based on the conversion of the tetrazolium salt WST-1 in a colored dye by cellular mitochondrial dehydrogenases enzymes. It measured the cells metabolic activity, which was proportional to the number of viable cells.

\subsection{In Vivo Biological Methods \\ Husbandry and Toxicity Assessment}

Adult zebrafish were maintained and treated as previously described [26]. Briefly, embryos at $4 \mathrm{~h}$ post fertilization (hpf) were gathered, washed several times with E3 medium, and exposed to different solutions of BN-CNOs or oxi-BN-CNOs $(5,10,50$, and $100 \mu \mathrm{g} / \mathrm{mL})$ until $120 \mathrm{hpf}$. The possible effects of BN-CNOs and oxi-BN-CNOs on the embryonic developmental stages were assessed in terms of the heart rate, swimming activity, malformation rate, hatching rate, and survival rate using a stereomicroscope armed with a digital camera at different hours post fertilization. Each experiment was repeated three times. All animal experiments were performed in full compliance with the revised directive 2010/63/EU.

\subsection{Statistical Analysis}

All data were expressed as mean \pm standard deviations of three replicates. Data were analyzed by one-way analysis of variance (ANOVA) in combination with Holm-Sidak post hoc test to compare each treated group with negative controls. A value of $p \leq 0.01$ (marked by an asterisk) was considered statistically significant. 


\section{Results and Discussion}

\subsection{Synthesis and Characterization of the CNO Materials}

The synthetic procedures employed for the production of boron/nitrogen doped carbon nano-onions (BN-CNOs) and its oxidized counterpart (oxi-BN-CNOs) are shown in Figure 1. The synthesis of $\mathrm{BN}-\mathrm{CNO}$ s was accomplished by thermally annealing a mixture of DNDs and boric acid $\left(\mathrm{H}_{3} \mathrm{BO}_{3}\right)$ at $1650{ }^{\circ} \mathrm{C}$ under a helium atmosphere [14], resulting in the efficient incorporation of the dopants into the carbon network. The modification of $\mathrm{BN}-\mathrm{CNO}$ via chemical oxidation to yield oxi-BN-CNOs was achieved by treating a $\mathrm{CNO}$ dispersion in $3 \mathrm{M}$ nitric acid $\left(\mathrm{HNO}_{3}\right)$ for $48 \mathrm{~h}$ under reflux conditions [3], resulting in a high coverage of the $\mathrm{BN}-\mathrm{CNO}$ surface with carboxylic acid groups.



Figure 1. Synthetic procedure for the production of BN-CNOs and oxi-BN-CNOs.

To cast light on the effects of the oxidation procedure, the morphological features of the $\mathrm{CNO}$ materials were investigated by HRTEM imaging. Figure 2A shows a representative HRTEM image of BN-CNOs, showing the existence of polyhedral CNOs as previously reported [14]. Following the oxidation procedure, no differences in the CNO morphology were observed (Figure 2C), suggesting that the process did not affect the integrity of the carbon structure.

EELS analyses were employed to obtain information on the chemical composition of the CNO derivatives. The EEL spectra of BN- and oxi-BN-CNOs are reported in Figure $2 B, D$, including the STEM images of the investigated areas for each sample, displayed as an insert.

The EELS analysis of BN-CNOs (Figure 2B) reveals the presence of the boron (B), carbon $(\mathrm{C})$, and nitrogen $(\mathrm{N}) \mathrm{K}$-edge structures. The distribution of these three elements indicates a mixture of both dopants in the carbon network, in line with what was previously reported [14]. Furthermore, the analysis of the fine structures near the edges (ELNES) for the $\mathrm{K}$ edges of $\mathrm{B}, \mathrm{C}$, and $\mathrm{N}$ revealed $\mathrm{sp}^{2}$-like phases, with a high crystalline quality [27-31].

In contrast, oxi-BN-CNOs exhibit a different behavior to that of BN-CNOs. Indeed, B, $\mathrm{C}$, and $\mathrm{N}$ are only present in the innermost layers with the outmost layers showing only the C-K edge (red and blue lines in Figure 2D and its inset, respectively). This could be due to the surface removal of the dopants as a consequence of the oxidation process.

In any case, from the ELNES analyses of the three K edges, we can also confirm that both areas (internal and external) of the CNOs show a very good "graphitic-like" quality.

The further confirmation of the removal of dopants from the $\mathrm{CNO}$ surface is provided by investigating the elemental composition of both samples by XPS. Figure 3A,E report the XPS survey spectra of BN-CNOs and their oxidized derivatives, displaying characteristic peaks due to the presence of carbon $(\mathrm{C})$, boron $(\mathrm{B})$, and nitrogen $(\mathrm{N})$, as well as oxygen $(\mathrm{O})$.

From the analysis of Table 1, it is clear that the oxidation procedure leads to a remarkable increase in the $\mathrm{O}$ content (from 4.6 to 12 at. \%) of the oxidized $\mathrm{CNO}$ sample at the expense of $\mathrm{B}$ and $\mathrm{N}$, whose content is drastically reduced (from 8 to 1.4 at. \% and from 5.4 to 0.3 at. \%, respectively), supporting the surface removal of the dopants as a consequence of the oxidation process. 

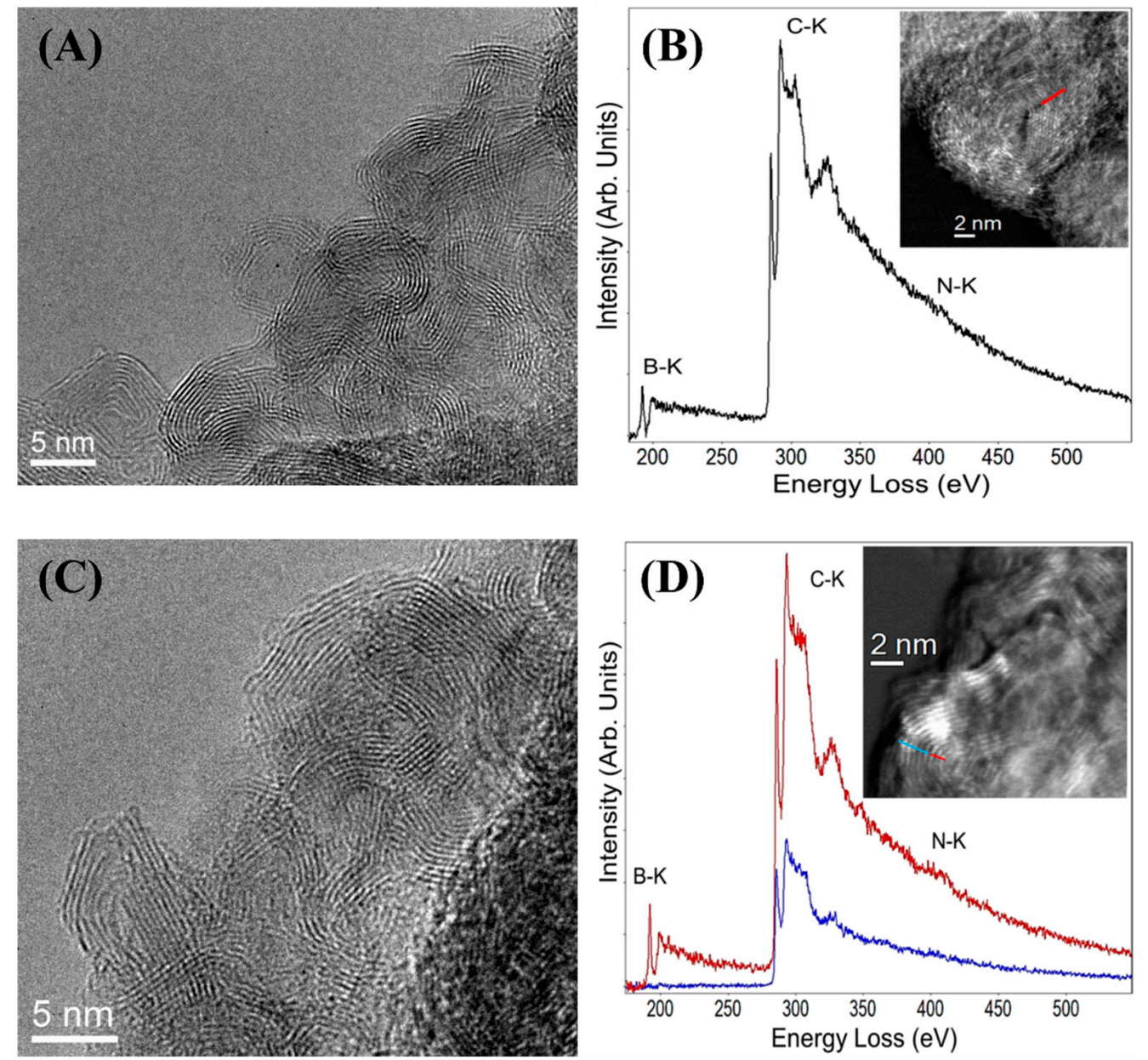

Figure 2. HRTEM images of BN-CNOs (A) and oxi-BN-CNOs (C), showing polyhedral-shaped CNOs. EELS spectra and corresponding STEM images as insets of BN-CNOs (B) and oxi-BN-CNOs (D), showing distinct B, C, and N-K edges. Blue and red lines in panel D refer to the external and internal layers, as depicted in the inset.

Table 1. Elemental compositions of BN- and oxi-BN-CNOs from XPS analyses.

\begin{tabular}{ccccc}
\hline Sample & C (at. \%) & O (at. \%) & B (at. \%) & N (at. \%) \\
\hline BN-CNOs & 82.0 & 4.6 & 8.0 & 5.4 \\
\hline oxi-BN-CNOs & 86.2 & 12.0 & 1.4 & 0.3 \\
\hline
\end{tabular}

We report that the introduction of both dopants into the CNO structure increases the defectiveness of the carbon network [14]. As the presence of B and $\mathrm{N}$ atoms enhances the chemical reactivity of the doped structures; therefore, they likely represent preferential sites for oxidation [32,33] and this can explain their lower content in the oxidized CNOs. In agreement with the EELS results, the modification of the CNO surface during the oxidation likely removes $\mathrm{B}$ and $\mathrm{N}$ from the external layers of the materials (with the latter to a great extent compared to the former) with the creation of $\mathrm{sp}^{3}$ hybridized carbon atoms. On the contrary, as the oxidation occurs preferentially on the CNO surface, the inner part is not affected by the procedure, showing a preserved doped nature. 

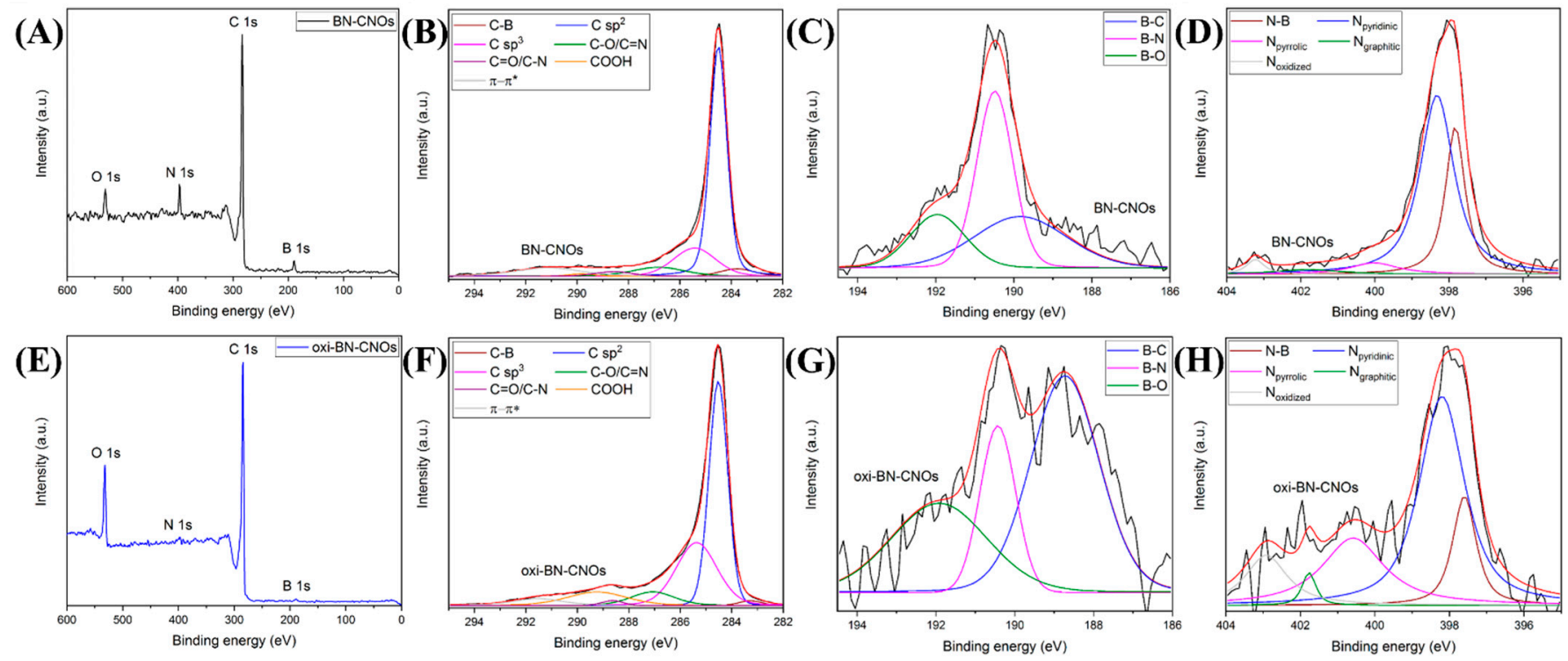

Figure 3. XPS survey (A,E) and high-resolution C 1s (B,F), B 1s (C,G), and N 1s (D,H) XPS spectra of BN-CNOs (upper panel) and oxi-BN-CNOs (lower panel), including peak deconvolution and experimental (black) and fitting curves (red).

Further information regarding the chemical states of the elements can be obtained from the analysis of the high-resolution C 1s, B 1s, and N 1s XPS spectra of BN- and oxi-BNCNOs, reported in Figure 3. Each spectrum, after a post-processing peak deconvolution process, is composed of different contributions reported in Tables 2 and 3.

Table 2. Chemical state, positions (eV), and relative area percentages of the deconvoluted C 1s peaks obtained from XPS analyses of BN- and oxi-BN-CNOs.

\begin{tabular}{ccccccccc}
\hline Sample & $\mathbf{C - B}$ & $\mathbf{C ~ s p}^{\mathbf{2}}$ & $\mathbf{C ~ s p}^{\mathbf{3}}$ & $\mathbf{C}-\mathbf{O} / \mathbf{C}=\mathbf{N}$ & $\mathbf{C}=\mathbf{O} / \mathbf{C}-\mathbf{N}$ & $\mathbf{C O O H}$ & $\boldsymbol{\pi}-\boldsymbol{\pi}^{*}$ \\
\hline \multirow{2}{*}{ BN-CNOs } & 283.7 & 284.5 & 285.4 & 286.8 & 288.6 & 289.8 & 291.1 \\
& $(3.4 \%)$ & $(59.6 \%)$ & $(17.4 \%)$ & $(7.1 \%)$ & $(2.5 \%)$ & $(0.9 \%)$ & $(9.1 \%)$ \\
\hline \multirow{2}{*}{ oxi-BN-CNOs } & 283.3 & 284.5 & 285.4 & 287.0 & 288.6 & 289.3 & 291.5 \\
& $(1.2 \%)$ & $(46.5 \%)$ & $(29.6 \%)$ & $(7.2 \%)$ & $(1.2 \%)$ & $(9.8 \%)$ & $(4.5 \%)$ \\
\hline
\end{tabular}

Table 3. Chemical state, positions (eV), and relative area percentages of the deconvoluted B 1s and $\mathrm{N}$ 1s peaks obtained from XPS analyses of BN- and oxi-BN-CNOs.

\begin{tabular}{ccccccccc}
\hline Sample & B-C & B-N & B-O & N-B & $\mathbf{N}_{\text {pyridinic }}$ & $\mathbf{N}_{\text {pyrrolic }}$ & $\mathbf{N}_{\text {graphitic }}$ & $\mathbf{N}_{\text {oxidized }}$ \\
\hline \multirow{2}{*}{ BN-CNOs } & 189.8 & 190.5 & 191.9 & 397.8 & 398.3 & 400.0 & 401.8 & 403.2 \\
& $(33.8 \%)$ & $(45.3 \%)$ & $(20.9 \%)$ & $(28.4 \%)$ & $(59.5 \%)$ & $(5.9 \%)$ & $(3.4 \%)$ & $(2.8 \%)$ \\
\hline \multirow{2}{*}{ oxi-BN-CNOs } & 188.7 & 190.4 & 191.9 & 397.6 & 398.2 & 400.6 & 401.8 & 402.9 \\
& $(51.2 \%)$ & $(19.45 \%)$ & $(29.4 \%)$ & $(13.4 \%)$ & $(49.1 \%)$ & $(23.3 \%)$ & $(2.4 \%)$ & $(11.8 \%)$ \\
\hline
\end{tabular}

The $\mathrm{C}$ 1s peak of BN-CNOs (Figure 3B) consists of seven different components [14]. The peak at $283.7 \mathrm{eV}$ is assigned to the C-B bonding state [34], while the two most intense peaks located at 284.5 and $285.4 \mathrm{eV}$ are attributed to carbon atoms with $\mathrm{sp}^{2}$ and $\mathrm{sp}^{3}$ hybridization, respectively. The other components at higher binding energies are ascribed to oxygen- and nitrogen-bonded carbon atoms [35,36] $(\mathrm{C}-\mathrm{O}$ and $\mathrm{C}=\mathrm{N}$ at $286.8 \mathrm{eV}$, $\mathrm{C}=\mathrm{O}$, and $\mathrm{C}-\mathrm{N}$ at $288.5 \mathrm{eV}$, as well as $\mathrm{COOH}$ at $289.8 \mathrm{eV}$ ) and the $\pi-\pi^{*}$ contribution $(291.1 \mathrm{eV})$. After the oxidation, the analysis of the C 1s spectrum of oxi-BN-CNOs, reported in Figure 3F, reveals a consistent increase in the peak corresponding to the $\mathrm{COOH}$ content, and thus confirming the preferential formation of the carboxylic acid functionalities onto the $\mathrm{CNO}$ surface. Moreover, the substantial enhancement of the $\mathrm{sp}^{3}$ character, together 
with the decrease in the nitrogen- and boron-bonded carbon states, is in agreement with the above-discussed EELS results. In addition, the intensity decrease in the $C=C$ bonds and $\pi-\pi^{*}$ contributions upon oxidation, which is ascribed to the photo-ionization of the $\pi$-electrons in conjugated systems [37], is in line with the introduction of defects into the graphitic nanostructures.

Additional insights on the effects of the oxidation on the chemical environment can be deduced by studying the differences between the $\mathrm{B} 1 \mathrm{~s}$ and $\mathrm{N}$ 1s peaks of $\mathrm{BN}$ CNOs (Figure 3C,D) and oxi-BN-CNOs (Figure 3G,H). The B 1s peak can be deconvoluted into three components assigned to B-C, B-N, and B-O bonding states $[14,38,39]$, which are located at 189.8, 190.5, and $191.9 \mathrm{eV}$, respectively. While in the pristine material the B-N species are predominantly present, the oxidation leads to a drastic reduction of these bonding states (i.e., more than half of the total content), suggesting a preferential consumption in favor of the carbon- and oxygen-bonded states (Table 3). Similar results can be observed from the analysis of the $\mathrm{N}$ 1s peak, which is composed of five different contributions located at 397.8, 398.3, 400.0, 401.8, and $403.2 \mathrm{eV}$, ascribed to N-B, pyridinic, pyrrolic, graphitic, and oxidized $\mathrm{N}$ types, respectively $[14,39,40]$. After the oxidation, in line with what was observed for the B 1s peak, a marked decrease in the N-B bonding states is detected upon oxidation together with a minor reduction of the pyridine and graphitic $\mathrm{N}$ atoms (Table 3). Therefore, the increase in the pyrrolic-like and oxidized $\mathrm{N}$ species strongly suggests that their formation is probably due to the oxidation of the former types of $\mathrm{N}$ atoms, as reported for other N-doped carbon nanostructures [33,41].

Therefore, from the XPS analyses, it can be deduced that the oxidation preferentially disrupts the B-N species, which are the predominant bond type in BN-CNOs, and this accounts for the marked decrease in the B and $\mathrm{N}$ contents in the oxidized counterparts.

UV-Vis absorption spectroscopy and DLS were employed to investigate the effects of the oxidation procedure on the water dispersibility of oxi-BN-CNOs.

Figure 4A,B show the UV-Vis spectra of BN- and oxi-BN-CNO dispersions in deionized water at different concentrations $(5-100 \mu \mathrm{g} / \mathrm{mL})$. Both samples exhibit absorption over the whole wavelength range with two absorption maxima, attributed to the $\pi \rightarrow \pi^{*}$ electronic transition of the $C=C$ bonds, located approx. at 200 and $260 \mathrm{~nm}$. Compared to that of BN$\mathrm{CNOs}$, the oxidized material displays much higher absorbance values at all investigated concentrations, thus suggesting a better water dispersibility of the oxidized derivatives ascribed to the increased hydrophilicity imparted by the introduction of carboxylic acid groups onto the material surface by the oxidative treatment.

Further evidence of the superior dispersion abilities of oxi-BN-CNOs is provided by the analysis of the CNO solutions reported in Figure 4C, showing darker colors for the oxidized CNO at the same concentration (bottom panel in Figure 4C), and thus confirming that the introduction of carboxylic acid groups onto the material surface provides an excellent water dispersibility to the oxidized derivatives.

Additional information about the dispersing abilities of both samples was obtained by DLS. Figure 5A shows the DLS spectra of the oxi-BN-CNO water dispersions at two representative concentrations ( 5 and $10 \mu \mathrm{g} / \mathrm{mL}$ ), showing the hydrodynamic diameters of 216.2 and $223.3 \mathrm{~nm}$, respectively. Although we were able to obtain the UV-Vis absorption spectra for BN-CNOs (Figure 4A), their dispersions were not homogeneous enough for assessing reliable DLS measurements due to the polydisperse nature of the particles caused by the heavy aggregation of the materials in water. On the contrary, as depicted in Table 4, it is evident that the oxidation treatment imparts a notable dispersing effect on oxi-BN-CNOs, as indicated by the hydrodynamic size values around $220 \mathrm{~nm}$ and negligible differences in the concentrations, thus confirming the superior dispersion in water to that of BN-CNOs. 

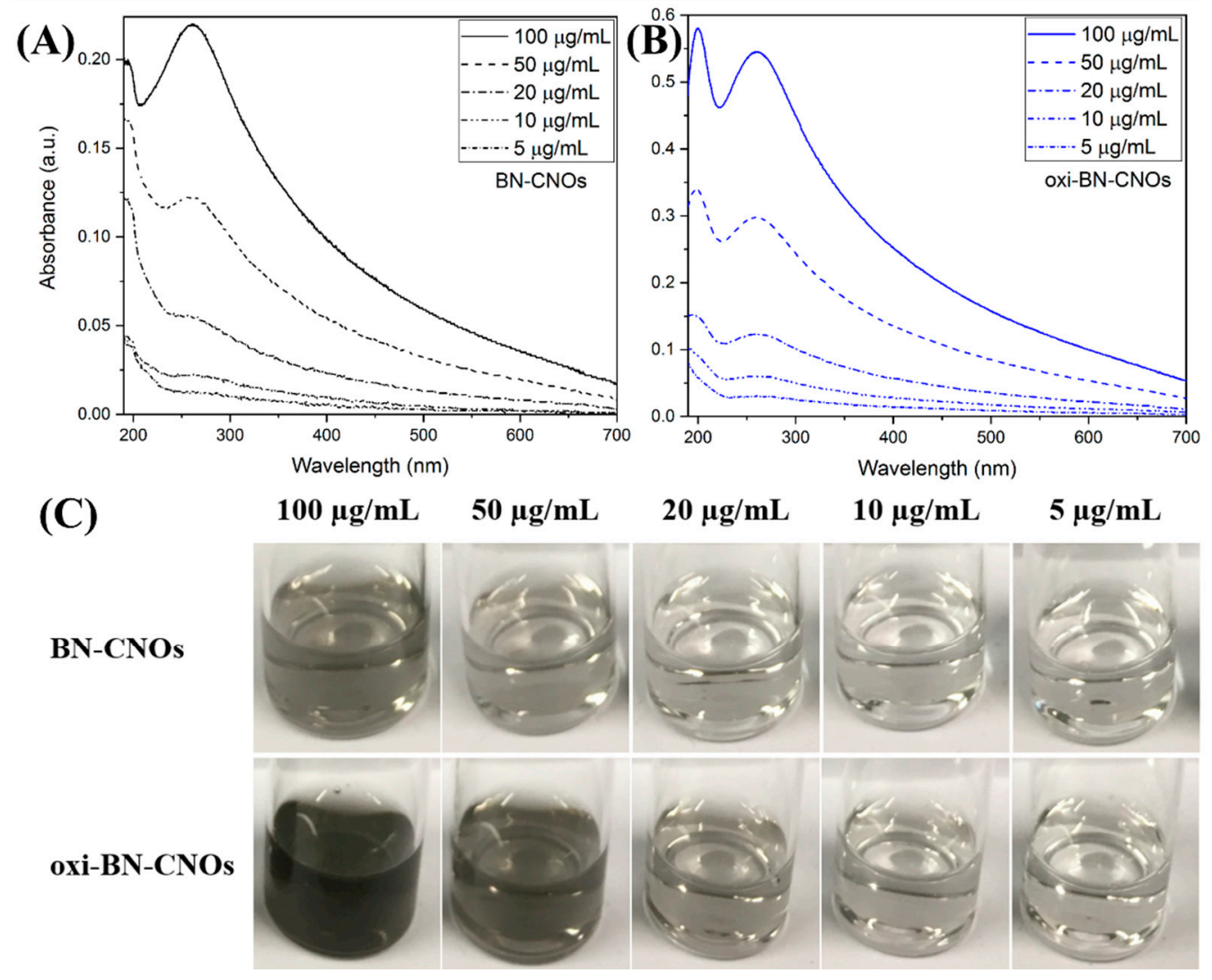

Figure 4. UV-Vis absorption spectra of (A) BN- and (B) oxi-BN-CNO dispersions in deionized water at different concentrations $(5,10,20,50$ and $100 \mu \mathrm{g} / \mathrm{mL})$. (C) Pictures of the solutions at the different concentrations.
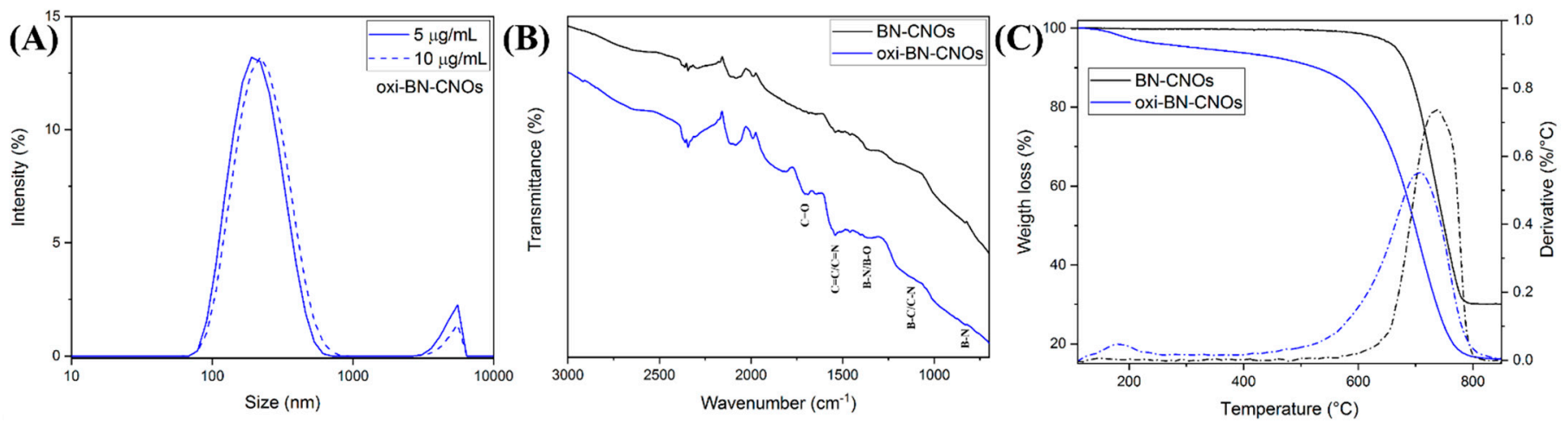

Figure 5. (A) DLS spectra of oxi-BN-CNO dispersions in deionized water at concentrations of 5 and $10 \mu \mathrm{g} / \mathrm{mL}$. (B) FTIR spectra and (C) TGA (solid lines) and corresponding weight loss derivatives (dotted lines) of BN-(black) and oxi-BNCNOs (blue).

Table 4. Hydrodynamic diameters of oxi-BN-CNOs at 5 and $10 \mu \mathrm{g} / \mathrm{mL}$ in deionized water from DLS analyses and weight loss (w.l.) at $450^{\circ} \mathrm{C}$, decomposition temperatures and residues at $850{ }^{\circ} \mathrm{C}$ of $\mathrm{BN}$ and oxi-BN-CNOs from TGA analyses.

\begin{tabular}{|c|c|c|c|c|c|}
\hline Sample & $\begin{array}{c}\text { Size @ } \\
5 \mu \mathrm{g} / \mathrm{mL}\end{array}$ & $\begin{array}{c}\text { Size @ } \\
10 \mu \mathrm{g} / \mathrm{mL}\end{array}$ & $\begin{array}{l}\text { w.1. @ } \\
450{ }^{\circ} \mathrm{C}\end{array}$ & $T_{D}$ & $\begin{array}{c}\text { Residue @ } \\
850^{\circ} \mathrm{C}\end{array}$ \\
\hline BN-CNOs & - & - & $0.3 \%$ & $736.0^{\circ} \mathrm{C}$ & $30.1 \%$ \\
\hline oxi-BN-CNOs & $216.2 \pm 12 \mathrm{~nm}$ & $223.3 \pm 8 \mathrm{~nm}$ & $7.3 \%$ & $706.0^{\circ} \mathrm{C}$ & $16.1 \%$ \\
\hline
\end{tabular}


Further confirmation of the successful surface modification of the co-doped CNOs was obtained by FTIR analyses, providing additional information regarding the functional groups introduced during the CNO surface modification (Figure $5 \mathrm{~B}$ ).

While the FTIR spectrum of BN-CNOs, reported in black in Figure 5B, displays typical peaks assigned to mixed $\mathrm{B}, \mathrm{C}$, and $\mathrm{N}$ bonding states [14], an additional peak at around $1700 \mathrm{~cm}^{-1}$ is observed in the oxi-BN-CNO FTIR spectrum (blue line in Figure 5B), which is attributed to the carbonyl group $(\mathrm{C}=\mathrm{O})$ stretching vibration modes. Therefore, the appearance of this new contribution confirms the formation of carboxylic acid functionalities over the $\mathrm{CNO}$ surface, in perfect agreement with what was observed from the analysis of the high-resolution C 1s XPS spectrum of oxi-BN-CNOs (Figure 3F).

Further confirmation of the successful surface modification of the co-doped CNOs was obtained by FTIR and TGA analyses. The analysis of the weight loss and derivative curves of both CNO materials, which is shown in Figure 5B, reveals that BN-CNOs exhibit a remarkable thermal stability up to $600^{\circ} \mathrm{C}$, when the decomposition of the carbon network starts occurring [14]. On the other hand, the oxidized CNOs exhibit a two-stage decomposition behavior. The first mass loss, occurring below $200^{\circ} \mathrm{C}$, is due to the degradation of the oxygen-containing groups, while the second is due to the decomposition of the carbon structure. The net weight loss at $450{ }^{\circ} \mathrm{C}$ (approx. $7 \%$ ) and the decreased decomposition temperature (approx. $30^{\circ} \mathrm{C}$ less than that of BN-CNOs), as shown in Table 4 , accounts for the introduction of defects as a consequence of the oxidation process, thus confirming the successful functionalization of the $\mathrm{CNO}$ surface. In addition, the lower mass residue at $850{ }^{\circ} \mathrm{C}$ reported for the oxidized derivate, attributed to the generation of boron oxides [14,42], further confirms the consumption of boron induced by the oxidative process.

Our characterizations confirm the successful formation of the oxidized doped CNOs, thus allowing us to investigate the biological behavior of these new members of the $\mathrm{CNO}$ family both in vitro and in vivo.

\subsection{In Vitro and In Vivo Studies}

To accurately define the cytotoxicity of the $\mathrm{CNO}$ materials on cells, we decided to employ both a healthy (mouse embryonic fibroblast cells, NIH 3T3) and a cancer cell line (human breast adenocarcinoma cells, MCF7). The possible effects of both CNOs on NIH 3T3 and MCF7 cells were assessed by means of the colorimetric WST1 assay. The two cell lines were incubated with BN- and oxi-BN-CNOs for 24, 48, and $72 \mathrm{~h}$.

Figure 6 displays the cell viability of NIH $3 \mathrm{~T} 3$ and MCF7 cells after the exposure at the same concentrations $(0.5,1,5$, and $10 \mu \mathrm{g} / \mathrm{mL})$ of the $\mathrm{CNO}$ materials.

The cell viabilities of NIH 3T3 and MCF7 cells were not affected after the treatment with the different dilutions of BN-CNOs (Figure 6A,B). NIH 3 T3 and MCF7cells treated with the highest dose of $\mathrm{BN}-\mathrm{CNO}$ showed cell viability values of $90 \%$ and $92 \%$, respectively, after $24 \mathrm{~h}$ of incubation. With a similar behavior, no inhibitory effects were observed upon NIH 3T3 and MCF7 cells exposed to oxi-BN-CNOs (Figure 6C,D) for all the treatments (0.5, 1,5 and $10 \mu \mathrm{g} / \mathrm{mL}$ ) and time points. NIH 3T3 and MCF7 cells treated with the highest dose of oxi-BN-CNOs showed cell viability values of $92 \%$ and $93 \%$, respectively, after $48 \mathrm{~h}$ of incubation. These results show a high cyto-biocompatibility of BN- and oxi-BN-CNOs in both healthy and cancer cells.

These findings are in agreement with our previous in vitro reports on the toxicological profile of CNOs. In fact, we previously reported a low toxicity or absence of toxicity of different $\mathrm{CNO}$ derivatives in different cell lines, including KB cells, HeLa Kyoto cells, human breast carcinoma cells (MDA-MB-231), and human ovarian carcinoma cells (A2780 cells) [2]. However, the newly reported doped CNOs seem to exhibit a higher biocompatibility with respect to the previously reported $\mathrm{CNO}$ materials. In particular, they present values of cell viabilities 5 to $10 \%$ higher than those shown by the other $\mathrm{CNO}$ derivatives at the highest concentrations tested (i.e., 5 and $10 \mu \mathrm{g} / \mathrm{mL}$ ). 
To corroborate the toxicological profile in the cells, the effects of the doped CNOs in zebrafish during the growth were assessed by treating fertilized embryos in solutions of BN-CNOs and oxi-CNOs at different dilutions $(5,10,50$, and $100 \mu \mathrm{g} / \mathrm{mL})$.

NIH 3 T3

(A)
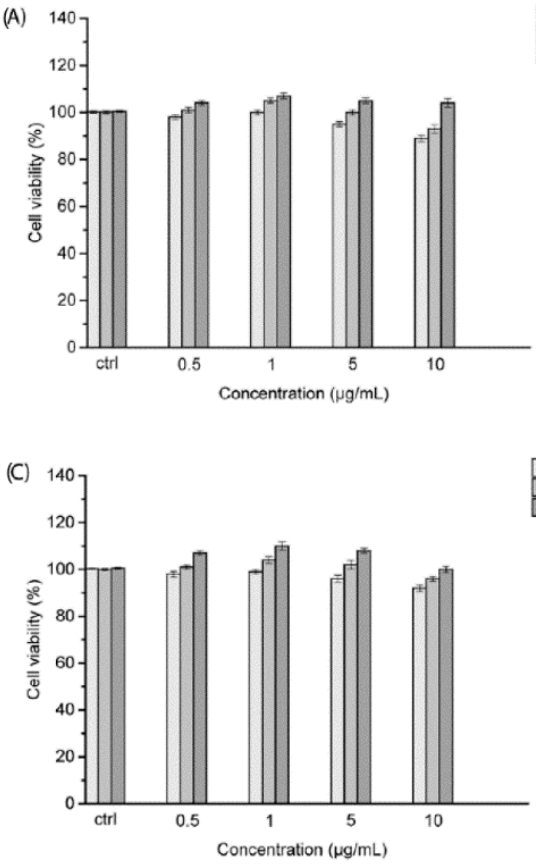

MCF7
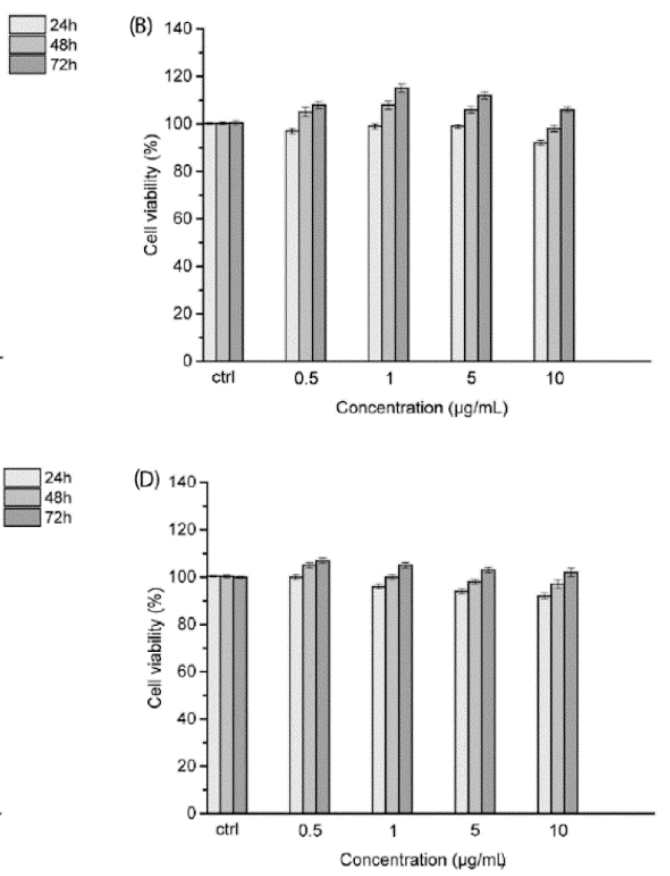

Figure 6. Cellular viability of NIH $3 \mathrm{~T} 3$ and MCF7 cells treated with various concentrations of BN(A,B) and oxi-BN-CNOs (C,D) for 24, 48, and $72 \mathrm{~h}$. Data are expressed as mean \pm standard deviations of three replicates.

Several toxicological endpoints were evaluated during the whole treatment (4-120 hpf), as reported in Figure 7. The survival rates of zebrafish embryos exposed to BN-CNOs, shown in Figure 7A, presented no significant changes in comparison to the control groups up to $50 \mu \mathrm{g} / \mathrm{mL}$, while they induced a significant change at $100 \mu \mathrm{g} / \mathrm{mL}$ after $120 \mathrm{hpf}$. On the other hand, the survival rates of zebrafish embryos exposed to oxi-BN-CNOs (Figure 7C) showed important changes only between 96 and $120 \mathrm{hpf}$ at a dose of $50 \mu \mathrm{g} / \mathrm{mL}$ and between 48 and $120 \mathrm{hpf}$ at a concentration of $100 \mu \mathrm{g} / \mathrm{mL}$. Similarly, the time of hatching was not affected by the exposure to BN-(Figure 7B) and oxi-BN-CNOs (Figure 7D), showing no inhibition in this parameter. These values of survival and hatching rates indicated a nontoxic profile of both materials, as per OECD guidelines (normative law).

In addition, we evaluated the heart rate of the exposed larvae, as shown in Figure 8. The average value of heartbeats showed no considerable changes compared to the controls (Figure 8A,C). Additionally, both BN-CNOs and oxi-BN-CNOs did not exert an influence on the swimming activity of treated larvae, with no consequent reduction/increase in the movement frequency with respect to the controls (Figure 8B,D).

The values of these toxicological endpoints demonstrated the biosafety of the $\mathrm{CNO}$ materials in the range of the investigated parameters. These findings are in agreement with the in vitro assessment in NIH 3T3 and MCF7 cells and with previously reported studies on the biocompatibility of different $\mathrm{CNO}$ derivatives in Danio rerio during the growth [5]. 

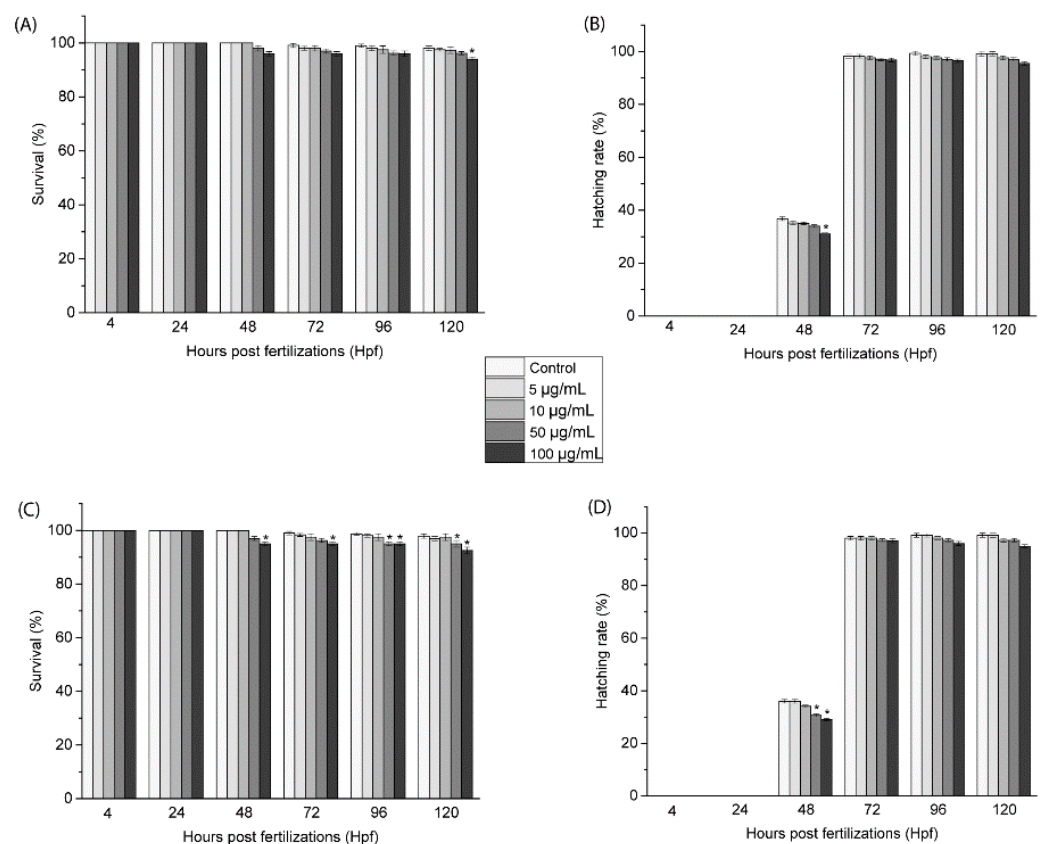

Figure 7. Survival rates (A-C) and hatching rates (B-D) of embryos exposed to different concentrations of BN- and oxi-BN-CNOs $(5,10,50$, and $100 \mu \mathrm{g} / \mathrm{mL})$. Data are calculated as means \pm S.D., from three independent experiments, $n=80$ ( ${ }^{*}$ indicate $p \leq 0.01$ compared to the control).
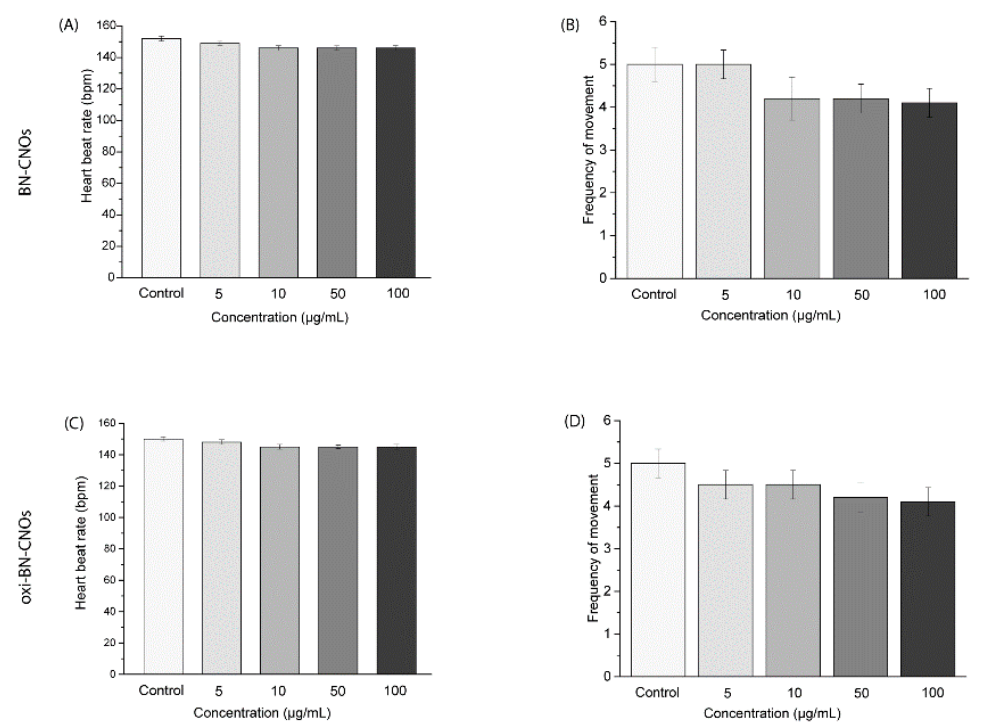

Figure 8. Heart rate (A-C) and frequency of voluntary movements (B-D) of larvae at 72 hpf exposed to different concentrations of $\mathrm{BN}$ - and oxi-BN-CNOs.

\section{Conclusions}

In this work, we evaluated for the first time the toxicological profile of a novel class of $\mathrm{CNO}$ materials, namely BN- and oxi-BN-CNOs. Our characterization techniques showed that the reported doping approach is effective in the introduction of both dopants onto the $\mathrm{CNO}$ surface, while the oxidation procedure is able to decorate the CNO surface with oxygen functionalities at the expense of the heteroatoms. This is attributed to the enhanced reactivity provided by the dopants that act as preferential sites.

Our in vitro, performed on both healthy and cancer cell lines, and in vivo studies confirm the low cytotoxicity and excellent biocompatibility of these novel $\mathrm{CNO}$ derivatives, opening the way for the exploration and use of the doped CNOs in biological applications. 
Author Contributions: S.G. conceived and designed the experiments; A.C. performed the synthesis, functionalization, and the XPS, UV-Vis, DLS, FTIR and TGA characterizations of BN-CNOs and oxi-BN-CNOs; R.A. performed the HRTEM, STEM and EELS analyses; M.d. performed the biological experiments; M.d. and A.C. wrote the paper. All authors contributed to the revisions of the paper. All authors have read and agreed to the published version of the manuscript.

Funding: This research was supported in part by the Spanish MICINN (project grant PID2019104739GB-100/AEI/10.13039/501100011033) from the Government of Aragon (project DGA E13-20R).

Institutional Review Board Statement: Not applicable.

Informed Consent Statement: Not applicable.

Data Availability Statement: The data presented in this study are available on request from the corresponding author.

Acknowledgments: The authors would like to acknowledge networking support by the COST Action CA19118 (High-performance Carbon-based Composites with Smart properties for Advanced Sensing Applications) and the European Union's Horizon 2020 program under the project "ESTEEM3" (823717), which made possible the HRTEM and EELS measurements performed in the Laboratorio de Microscopias Avanzadas (LMA) at the Universidad de Zaragoza (Spain). A.C. and S.G. wish to acknowledge the support from the School of Chemical Sciences. We also wish to thank Micaela Castellino (IIT) for assistance with the XPS measurements and Michał Bartkowski (DCU) for proofreading.

Conflicts of Interest: The authors declare no conflict of interest.

\section{References}

1. Ugarte, D. Curling and closure of graphitic networks under electron-beam irradiation. Nature 1992, 359, 707-709. [CrossRef] [PubMed]

2. Camisasca, A.; Giordani, S. Carbon nano-onions in biomedical applications: Promising theranostic agents. Inorg. Chim. Acta 2017, 468, 67-76. [CrossRef]

3. Lettieri, S.; Camisasca, A.; d'Amora, M.; Diaspro, A.; Uchida, T.; Nakajima, Y.; Yanagisawa, K.; Maekawa, T.; Giordani, S. Far-red fluorescent carbon nano-onions as a biocompatible platform for cellular imaging. RSC Adv. 2017, 7, 45676-45681. [CrossRef]

4. d'Amora, M.; Maffeis, V.; Brescia, R.; Barnes, D.; Scanlan, E.; Giordani, S. Carbon Nano-Onions as Non-Cytotoxic Carriers for Cellular Uptake of Glycopeptides and Proteins. Nanomaterials 2019, 9, 1069. [CrossRef]

5. d'Amora, M.; Camisasca, A.; Lettieri, S.; Giordani, S. Toxicity Assessment of Carbon Nanomaterials in Zebrafish during Development. Nanomaterials 2017, 7, 414. [CrossRef] [PubMed]

6. Frasconi, M.; Marotta, R.; Markey, L.; Flavin, K.; Spampinato, V.; Ceccone, G.; Echegoyen, L.; Scanlan, E.M.; Giordani, S. Multi-Functionalized Carbon Nano-onions as Imaging Probes for Cancer Cells. Chemistry 2015, 21, 19071-19080. [CrossRef] [PubMed]

7. Giordani, S.; Camisasca, A.; Maffeis, V. Carbon Nano-onions: A Valuable Class of Carbon Nanomaterials in Biomedicine. Curr. Med. Chem. 2019, 26, 6915-6929. [CrossRef]

8. Camisasca, A.; Giordani, S. Carbon Nano-onions for Bioimaging and Cancer Therapy Applications. In Nanooncology: Engineering Nanomaterials for Cancer Therapy and Diagnosis; Gonçalves, G., Tobias, G., Eds.; Springer International Publishing: Cham, Switzerland, 2018; pp. 417-455.

9. Bartkowski, M.; Giordani, S. Carbon nano-onions as potential nanocarriers for drug delivery. Dalton Trans. 2021, 50, 2300-2309. [CrossRef]

10. Breczko, J.; Plonska-Brzezinska, M.E.; Echegoyen, L. Electrochemical oxidation and determination of dopamine in the presence of uric and ascorbic acids using a carbon nano-onion and poly(diallyldimethylammonium chloride) composite. Electrochim. Acta 2012, 72, 61-67. [CrossRef]

11. Bartolome, J.P.; Echegoyen, L.; Fragoso, A. Reactive Carbon Nano-Onion Modified Glassy Carbon Surfaces as DNA Sensors for Human Papillomavirus Oncogene Detection with Enhanced Sensitivity. Anal. Chem. 2015, 87, 6744-6751. [CrossRef]

12. Mohapatra, J.; Ananthoju, B.; Nair, V.; Mitra, A.; Bahadur, D.; Medhekar, N.V.; Aslam, M. Enzymatic and non-enzymatic electrochemical glucose sensor based on carbon nano-onions. Appl. Surf. Sci. 2018, 442, 332-341. [CrossRef]

13. Cumba, L.R.; Camisasca, A.; Giordani, S.; Forster, R.J. Electrochemical Properties of Screen-Printed Carbon Nano-Onion Electrodes. Molecules 2020, 25, 3884. [CrossRef]

14. Camisasca, A.; Sacco, A.; Brescia, R.; Giordani, S. Boron/Nitrogen-Codoped Carbon Nano-Onion Electrocatalysts for the Oxygen Reduction Reaction. ACS Appl. Nano Mater. 2018, 1, 5763-5773. [CrossRef]

15. Yuan, X.; Zhang, X.; Sun, L.; Wei, Y.; Wei, X. Cellular Toxicity and Immunological Effects of Carbon-based Nanomaterials. Part. Fibre Toxicol. 2019, 16, 18. [CrossRef] 
16. Garriga, R.; Herrero-Continente, T.; Palos, M.; Cebolla, V.L.; Osada, J.; Muñoz, E.; Rodríguez-Yoldi, M.J. Toxicity of Carbon Nanomaterials and Their Potential Application as Drug Delivery Systems: In Vitro Studies in Caco-2 and MCF-7 Cell Lines. Nanomaterials 2020, 10, 1617. [CrossRef] [PubMed]

17. Jović, D.; Jaćević, V.; Kuča, K.; Borišev, I.; Mrdjanovic, J.; Petrovic, D.; Seke, M.; Djordjevic, A. The Puzzling Potential of Carbon Nanomaterials: General Properties, Application, and Toxicity. Nanomaterials 2020, 10, 1508. [CrossRef]

18. OECD. Test No. 236: Fish Embryo Acute Toxicity (FET) Test; OECD Publishing: Paris, France, 2013.

19. Peterson, R.T.; MacRae, C.A. Systematic Approaches to Toxicology in the Zebrafish. Annu. Rev. Pharmacol. Toxicol. 2012, 52, 433-453. [CrossRef] [PubMed]

20. MacRae, C.A.; Peterson, R.T. Zebrafish as tools for drug discovery. Nat. Rev. Drug. Discov. 2015, 14, 721-731. [CrossRef] [PubMed]

21. McCollum, C.W.; Ducharme, N.A.; Bondesson, M.; Gustafsson, J.-A. Developmental toxicity screening in zebrafish. Birth Defects Res. Part C Embryo Today Rev. 2011, 93, 67-114. [CrossRef] [PubMed]

22. Blechinger Scott, R.; Warren James, T.; Kuwada John, Y.; Krone Patrick, H. Developmental toxicology of cadmium in living embryos of a stable transgenic zebrafish line. Environ. Health Perspect. 2002, 110, 1041-1046. [CrossRef]

23. Truong, L.; Harper, S.L.; Tanguay, R.L. Evaluation of Embryotoxicity Using the Zebrafish Model. In Drug Safety Evaluation: Methods and Protocols; Gautier, J.-C., Ed.; Humana Press: Totowa, NJ, USA, 2011; pp. 271-279.

24. Howe, K.; Clark, M.D.; Torroja, C.F.; Torrance, J.; Berthelot, C.; Muffato, M.; Collins, J.E.; Humphray, S.; McLaren, K.; Matthews, L.; et al. The zebrafish reference genome sequence and its relationship to the human genome. Nature 2013, 496, 498-503. [CrossRef]

25. d'Amora, M.; Giordani, S. Zebrafish Models of Nanotoxicity: A Comprehensive Account. In Nanomaterial Biointeractions at the Cellular, Organismal and System Levels; Sharma, N., Sahi, S., Eds.; Springer International Publishing: Cham, Switzerland, 2021; pp. 53-72.

26. d'Amora, M.; Lamberti, A.; Fontana, M.; Giordani, S. Toxicity assessment of laser-induced graphene by zebrafish during development. J. Phys. Mater. 2020, 3, 034008. [CrossRef]

27. Arenal, R.; de la Peña, F.; Stéphan, O.; Walls, M.; Tencé, M.; Loiseau, A.; Colliex, C. Extending the analysis of EELS spectrumimaging data, from elemental to bond mapping in complex nanostructures. Ultramicroscopy 2008, 109, 32-38. [CrossRef] [PubMed]

28. Arenal, R.; Lopez-Bezanilla, A. In Situ Formation of Carbon Nanotubes Encapsulated within Boron Nitride Nanotubes via Electron Irradiation. ACS Nano 2014, 8, 8419-8425. [CrossRef] [PubMed]

29. Arenal, R.; March, K.; Ewels, C.P.; Rocquefelte, X.; Kociak, M.; Loiseau, A.; Stéphan, O. Atomic Configuration of Nitrogen-Doped Single-Walled Carbon Nanotubes. Nano Lett. 2014, 14, 5509-5516. [CrossRef] [PubMed]

30. Arenal, R.; Blase, X.; Loiseau, A. Boron-nitride and boron-carbonitride nanotubes: Synthesis, characterization and theory. Adv. Phys. 2010, 59, 101-179. [CrossRef]

31. Arenal, R.; Stephan, O.; Cochon, J.-L.; Loiseau, A. Root-Growth Mechanism for Single-Walled Boron Nitride Nanotubes in Laser Vaporization Technique. J. Am. Chem. Soc. 2007, 129, 16183-16189. [CrossRef]

32. Cruz-Silva, R.; Morelos-Gómez, A.; Vega-Díaz, S.; Tristán-López, F.; Elias, A.L.; Perea-López, N.; Muramatsu, H.; Hayashi, T.; Fujisawa, K.; Kim, Y.A.; et al. Formation of Nitrogen-Doped Graphene Nanoribbons via Chemical Unzipping. ACS Nano 2013, 7, 2192-2204. [CrossRef]

33. Arkhipova, E.A.; Ivanov, A.S.; Strokova, N.E.; Chernyak, S.A.; Shumyantsev, A.V.; Maslakov, K.I.; Savilov, S.V.; Lunin, V.V. Structural evolution of nitrogen-doped carbon nanotubes: From synthesis and oxidation to thermal defunctionalization. Carbon 2017, 125, 20-31. [CrossRef]

34. Lin, Y.; Wu, S.; Shi, W.; Zhang, B.; Wang, J.; Kim, Y.A.; Endo, M.; Su, D.S. Efficient and highly selective boron-doped carbon materials-catalyzed reduction of nitroarenes. Chem. Commun. 2015, 51, 13086-13089. [CrossRef]

35. Choi, E.Y.; Kim, C.K. Fabrication of nitrogen-doped nano-onions and their electrocatalytic activity toward the oxygen reduction reaction. Sci. Rep. 2017, 7, 4178. [CrossRef] [PubMed]

36. Ratso, S.; Kruusenberg, I.; Vikkisk, M.; Joost, U.; Shulga, E.; Kink, I.; Kallio, T.; Tammeveski, K. Highly active nitrogen-doped few-layer graphene/carbon nanotube composite electrocatalyst for oxygen reduction reaction in alkaline media. Carbon 2014, 73, 361-370. [CrossRef]

37. Ismagilov, Z.R.; Shalagina, A.E.; Podyacheva, O.Y.; Ischenko, A.V.; Kibis, L.S.; Boronin, A.I.; Chesalov, Y.A.; Kochubey, D.I.; Romanenko, A.I.; Anikeeva, O.B.; et al. Structure and electrical conductivity of nitrogen-doped carbon nanofibers. Carbon 2009, 47, 1922-1929. [CrossRef]

38. Kim, S.Y.; Park, J.; Choi, H.C.; Ahn, J.P.; Hou, J.Q.; Kang, H.S. X-ray Photoelectron Spectroscopy and First Principles Calculation of BCN Nanotubes. J. Am. Chem. Soci. 2007, 129, 1705-1716. [CrossRef] [PubMed]

39. Jin, J.; Pan, F.; Jiang, L.; Fu, X.; Liang, A.; Wei, Z.; Zhang, J.; Sun, G. Catalyst-Free Synthesis of Crumpled Boron and Nitrogen Co-Doped Graphite Layers with Tunable Bond Structure for Oxygen Reduction Reaction. ACS Nano 2014, 8, 3313-3321. [CrossRef]

40. Baik, S.; Lee, J.W. Effect of boron-nitrogen bonding on oxygen reduction reaction activity of BN Co-doped activated porous carbons. RSC Adv. 2015, 5, 24661-24669. [CrossRef]

41. Achour, A.; Vizireanu, S.; Dinescu, G.; Le Brizoual, L.; Djouadi, M.A.; Boujtita, M. Electrochemical anodic oxidation of nitrogen doped carbon nanowall films: X-ray photoelectron and Micro-Raman spectroscopy study. Appl. Surf. Sci. 2013, 273, 49-57. [CrossRef]

42. Yuge, R.; Bandow, S.; Yudasaka, M.; Toyama, K.; Iijima, S.; Manako, T. Boron- and nitrogen-doped single-walled carbon nanohorns with graphite-like thin sheets prepared by $\mathrm{CO}_{2}$ laser ablation method. Carbon 2017, 111, 675-680. [CrossRef] 